\title{
Dynamic layout and optimization design of industrial safety fence
}

\author{
Chen Chen ${ }^{1, a}$, Peng Huang ${ }^{1, b, *}$ and Xiaoli Liu ${ }^{2, c}$ \\ ${ }^{1}$ YANZHOU COAL MINING CO.,LTD, The high-tech zone street agency of jining, Jining, 272000, \\ Shandong, China \\ ${ }^{2}$ School of Mechanical \& Automotive Engineering, Qilu University of Technology, Jinan, Shandong, 250353, \\ China \\ ${ }^{3}$ Electrical Engineering and the Automatization Specialty, Shandong University of Science and Technology, \\ Taian, Shandong, 271019, China

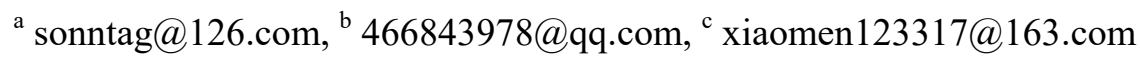

Keywords: Protect fence, Reasonable layout, Iterative method, optimal choice

\begin{abstract}
For achieving the standardized management of safety protective fence of mechanical equipment, regulating the arrangement of fence sizes, and solving the problems of waste materials of site fences and difficulties in trepanning fences, an optimized designing new program for the arrangement and trepanning of safety protective fences has been proposed. First of all, according to the mathematical model of optimization design clear fence stochastic dynamic layout constraints, in view of the objective function to achieve fence sorting simulation modeling. Then, based on the iterative random combination algorithm, combined with high-level language $\mathrm{c}++$, using object-oriented programming ideas, design a dynamic industrial protective enclosures decorate a process framework, finally, the algorithm introduced enterprise ERP system, the algorithm in the Volvo, faw Volkswagen robot application in the design of press line protection fence showed that after optimization design product costs an average of more than $10 \%$; Length of $700 \mathrm{~mm}, 800 \mathrm{~mm}$, $1000 \mathrm{~mm}$ fencing three kinds of specifications size error rate control within $1 \%$.
\end{abstract}

\section{Introduction}

Security fence is the most widely used in the production of industrial safety protection facilities, and application in the stamping production line, machine equipment, and other large industrial production, especially for multiple degrees of freedom robot production line, the safety fence will work and non-work area reasonable segmentation, prevent the robotic arms for injured workers, effectively reduce the occurrence of safety accidents [1]. For sorting is not in conformity with the requirements of a fence to conduct site cutting, because every time arrangement is different, different size, cutting parts to separate production, the high cost of each cutting accessories. Security fence difficult selection, equipment is difficult to determine the order and open hole position, special processing method of the fence is difficult problems become a big problem in the production enterprises, if improper handling can raise safety fence production cost, also can cause casualties because the device used is not reasonable, production safety accidents.

Dynamic layout problems for safety protection fence and hole problem, considering the use of fence quantity, the dynamic opening arrangement, put the order, fence permissible error factors, such as to avoid opening position and safety protection fence post intervention. In order to reduce the engineering technicians repeated multifarious workload and improve the efficiency of factory production, this paper proposes a optimization method of fence [2], a solution is given, and from the perspective of application, using $\mathrm{c}^{++}$language to write a useful program [3], analyzed data comparison practical cases. 


\section{Safety fence industry requirements}

Fig.1 A sliding door is provided in the enclosure. A is A special size, which needs to be customized or field cut, plus cutting accessories; figure 2 is the equipment working hole, through the working hole operation equipment. The installation clearance and baffle of the protective fence and column shall be considered separately in the width of each fence. The existing three lengths of protection fence $\mathrm{H}=\{\mathrm{a}, \mathrm{b}, \mathrm{c}\}$. The protection column width is 1 , the target length is 1 , the opening width is epsilon, the opening number is $\mathrm{N}$. The design protection fence is arranged and the opening position, so that the protection fence can be ordered to meet the target length[4-6].

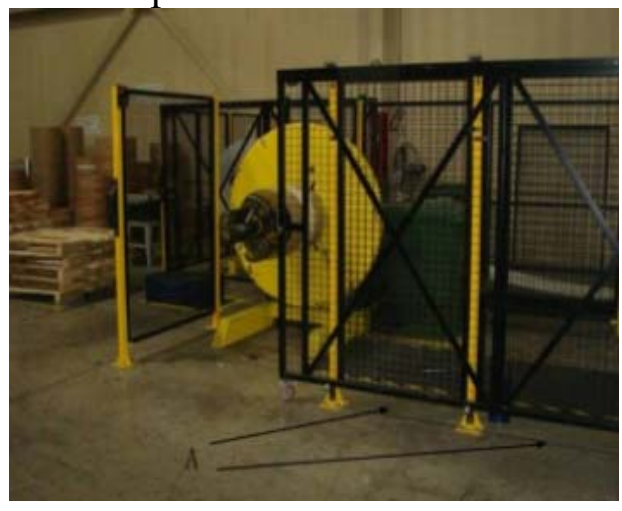

Figure 1 Special fence

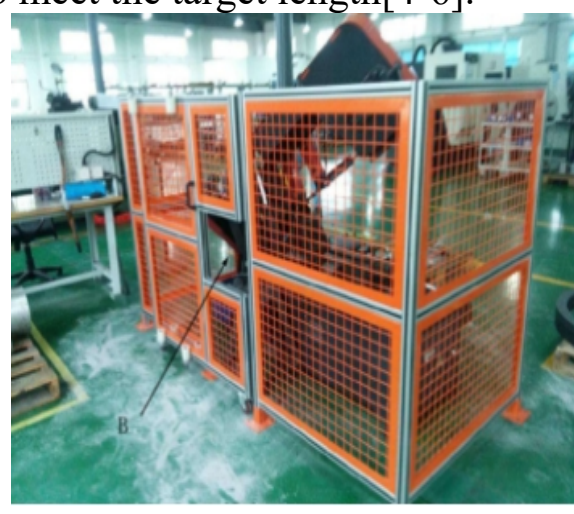

Figure 2 The Opening fence

\subsection{No work barriers are lined up}

\section{- The design variables}

No work hole protection fence arrangement, length L, the length of the three fence units is a, b, c, the width of the protective column is equal to $\mathrm{L}$, the allowable error range is $\mathrm{I}$, the maximum permutations of the three enclosures are max (a), max (b), max (c), x, y, z as design variables.

$$
X=[\mathrm{L}, \mathrm{l}, \mathrm{i}, \mathrm{a}, \mathrm{b}, \mathrm{c}]^{\mathrm{T}}=[x, \mathrm{y}, \mathrm{z}]^{\mathrm{T}}
$$

\section{- The objective function}

The length $\mathrm{L}$ is the main indicator of unit length arrangement. After determining $\mathrm{L}$, the minimum value greater than $\mathrm{L}$ is the goal that should be pursued, $\mathrm{a}, \mathrm{b}, \mathrm{c}$ three lengths of protection fence are arranged to get a minimum of $\mathrm{L}$. The design objective function of the fence is:

$$
\min f(x, y, z)=(a+1) * x+(b+1) * y+(c+1) * z
$$

\section{- The constraint condition}

In order to meet the practical requirements, some constraints must be set. Limit the upper and lower bounds of variables [7]:

$$
\left\{\begin{array}{l}
\mathrm{i}<\min (a, b, c) \\
0<\mathrm{i}<40 \\
1<\mathrm{a}<\max (\mathrm{a}) \\
1<\mathrm{b}<\max (\mathrm{b}) \\
1<\mathrm{c}<\max (\mathrm{c})
\end{array}\right.
$$

\section{- An optimization method}

A is the recommended size of three sizes. The recommended size is preferred in the first layer loop, the initial cycle is full of type a. If the error doesn't satisfy the constraint, in the next layer loop iterate over the growth of $b$, c, and the number of protective enclosures, rerun routine. After the three-tier loop sort is finished, the external constraint is still not satisfied, the error grows, and we continue to 
run the triple cycle, until there is a minimum that meets all the constraints, the iteration automatically stops and outputs the minimum. The column width and error and the 1 . The opening width is $\varepsilon$. The number of open holes is $\beta$. The length of the remainder of the opening hole is $b$.

$$
X=[L, d, l, \varepsilon, \beta, a, b, c]^{T}=\left[x, y, z, x_{1} \cdots X_{\beta}\right]^{T}
$$

\subsection{Arrangement of fences with working holes}

\section{- design variable}

According to the different requirements of the mechanical equipment, the fence needs to be controlled dynamically. A hole is arranged in the specified location, working hole size does not exceed 1.2 times the maximum length of the fence, In order to ensure the strength of the safety fence, the position of the work hole cannot be interfered with the vertical column. It is difficult to determine the scene of the machine, so reduce the number of site cut and the length. $L$ is the total length of the fence.

\section{- The objective function}

This objective function is divided into four stages according to the opening position.

Starting from the beginning, the opening position is the end point.

$$
\min f(x, y, z)=(a+l) * x+(b+l) * y+(c+l) * z
$$

The beginning of the opening is the beginning of the opening. The end point is the end of the opening.

$\min f(x, y, z)=d+l+\varepsilon<\max (a, b, c)$

The distance between the two openings.

$$
\min f(x, y, z)=(a+l) * x+(b+l) * y+(c+l) * z
$$

\section{- The constraint condition}

In order to meet the practical requirements, some constraints must be set. Limit the upper and lower bounds of variables:

$$
\left\{\begin{array}{l}
\mathrm{i}<\min (a, b, c) \\
0<i<40 \\
1<a<\max a \\
1<b<\max b \\
1<c<\max c \\
0<\varepsilon, d, d_{1}<\max (a, b, c) \\
x_{1}, x_{2} \cdots x_{\beta} \neq x_{l}
\end{array}\right.
$$

\section{- An optimization method}

The working hole protection fence calculation program is shown in figure 4.

After entering the length of the fence and the position of the hole, the operation of block diagram 3 is advanced, residual distance $\mathrm{d}$. The remaining distance $\mathrm{d}$, column width 1, opening size and the length of the three fences are compared. If the size of the fence is greater than the sum of the three sizes, the position of the post and the second opening of the fence shall be determined, if no interference, there is no cutting at the front of the first opening, the first opening is optimal in the size of the fence. In case of interference, the starting point of the second opening is the first opening, and the end point of the fence, no cutting at the opening, cut the front opening of the first opening to meet the requirement. The middle opening section is recalculated according to block diagram 3 until all the opening segments end. Remaining distance running program block diagram 3 , if there is a cutting 
distance, the cutting distance is incorporated into the adjacent opening section, the length of the original, the sum of the remaining distances and the comparison of the fences, if it is less than the length of the fence, the optimal output is obtained. If it is greater than the length of the fence, there is no cutting near the opening section, and the remaining distance is cut to meet the requirements[9-10].

\section{Fence design}

\subsection{Safety fence for machining workshop}

Take Volvo multi degree manipulator workshop as an example, different workshops are separated by safety fence. The length of the flame cutting zone is $20 \mathrm{~m}$, the width is 28 meters, and there is a square gap of $9 \mathrm{~m}^{*} 9 \mathrm{~m}$, the working length of the compressor is 28 meters, and the width is 8 meters

\subsection{Inspection of passing rate of fencing fence}

In actual production, the safety fence does not exceed the production workshop, and the length of the international standard factory building is generally less than $100 \mathrm{~m}$, according to the actual situation, given the length of the different security fence units, the same column width and gap interval requirements, the length of the fence within the same range is traversed. In the three case, the starting length is different, and the end length is $1234.56 \mathrm{~m}$.

- Case 1: There are three kinds of fencing fence, $700 \mathrm{~mm}, 800 \mathrm{~mm}, 1000 \mathrm{~mm}$, the width of the column is 7 , the starting length is 2320 .

- Case 2: There are four kinds of fencing fence, $200 \mathrm{~mm}, 500 \mathrm{~mm}, 700 \mathrm{~mm}, 1000 \mathrm{~mm}$, the width of the column is 7 , the starting length is 2400 .

- Case 3: There are two kinds of fencing fence $, 500 \mathrm{~mm}, 1000 \mathrm{~mm}$, the width of the column is 7 , the starting length is 1500 .

According to international regulations, the protection bar height error is within the range of $\pm 2 \mathrm{~cm}$, which is a qualified product.

In case 1, the traversal procedure can be seen in Figure 3.

Table 1 Case1 error range table

\begin{tabular}{c|ccccc} 
range of error & 0 & 1 & 2 & 3 & 4 \\
Interval number & 122077 & 565 & 321 & 75 & 45 \\
\hline range of error & 5 & 6 & 7 & 8 & 9 \\
Interval number & 45 & 45 & 17 & 17 & 17
\end{tabular}

Table 2 Case2 error range table

\begin{tabular}{c|ccc} 
range of error & 0 & 1 & 2 \\
\hline Interval number & 123210 & 4 & 2
\end{tabular}




\section{Table 3 Case 2 error range table}

\begin{tabular}{c|ccccccc} 
range of error & 0 & 1 & 2 & 3 & 4 & 5 & 6 \\
Interval number & 120484 & 732 & 612 & 492 & 372 & 252 & 132 \\
\hline range of error & 7 & $8-14$ & $15-21$ & $22-28$ & $29-35$ & $36-42$ \\
Interval number & 13 & $11 * 7=77$ & $8 * 7=56$ & $6 * 7=42$ & $4 * 7=28$ & $2 * 7=14$
\end{tabular}

The ratio of three different dimensions of safety fence is $99.89 \%, 100 \%, 99.50 \%$, the three kinds of dimensions meet the actual requirements of the project. The optimized safety fence makes the waste rate less than $1 \%$, reduce the on-site cutting process, save the working hours, reduce the waste and reduce the cost of the protection fence.

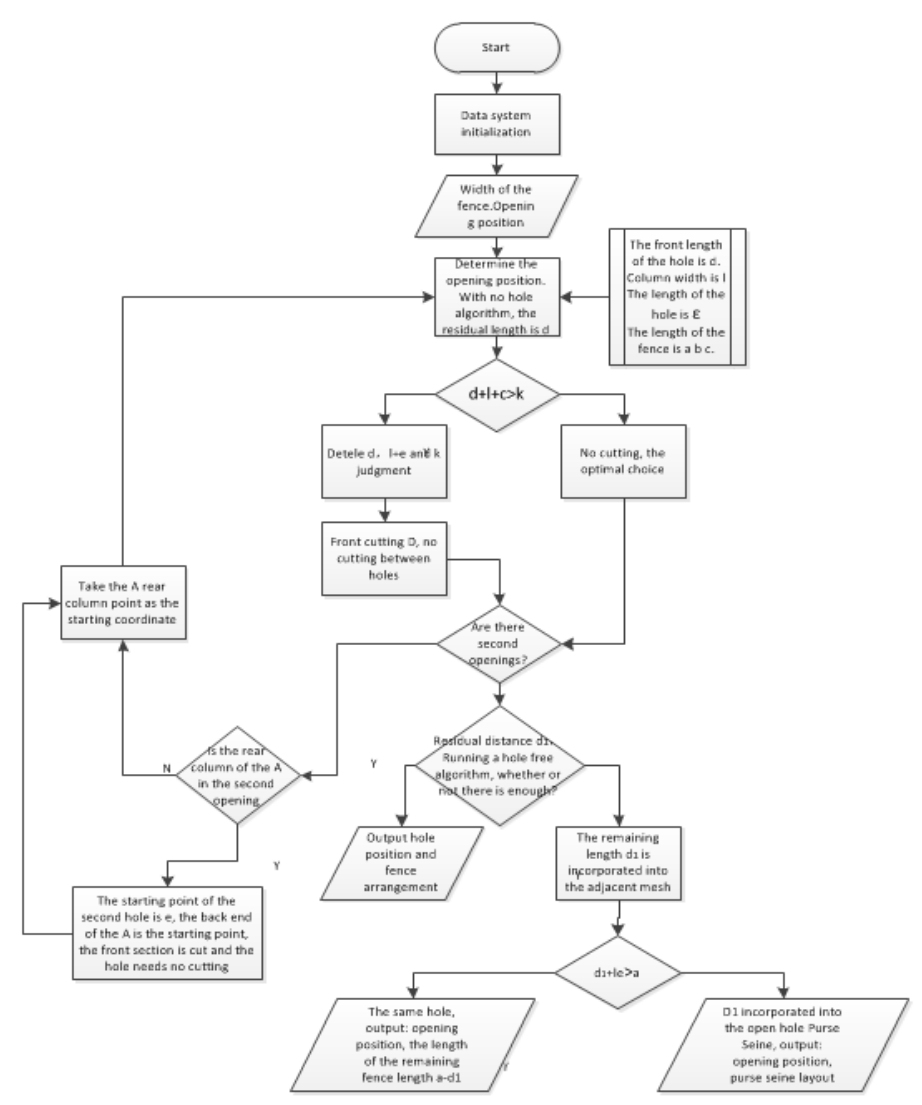

Figure 3 The hole arrangement block diagram

Step1: select wc,count $(*)$ from [ShieldAidDB].[dbo].[td_wlist] where len $>231$ group by wc order by wc

Step2: select count $(*)$ from [ShieldAidDB].[dbo].[td_wlist] where len $>231$

Step3: select * from [ShieldAidDB].[dbo].[td_wlist] where len $<232$

\subsection{Real cost reduction}

The use length is $700 \mathrm{~mm}, 800 \mathrm{~mm}, 1000 \mathrm{~mm}$ round net is arranged, the column width $7 \mathrm{~mm}$, respectively use figure 3 process and unoptimized design the arrangement of to compare. At least one field cut is required for each side of the normal arrangement. In addition to the cost of the fence itself, consideration should be given to on-site labour costs, installation of cutting parts (custom-made 
non-standard parts) costs.

Table 4 Optimize design materials use table

\begin{tabular}{c|cccc|cccc} 
& \multicolumn{4}{|c|}{ The original design } & \multicolumn{5}{c}{ Optimize design } \\
\hline $700 \mathrm{~mm}$ & \multicolumn{1}{|c}{} & & & 7 & 1 & 0 & 1 \\
$800 \mathrm{~mm}$ & & & & 3 & 7 & 7 & 19 \\
$1000 \mathrm{~mm}$ & 8 & 9 & 19 & 27 & & 2 & 13 & 10 \\
$7 \mathrm{~mm}$ & 8 & 9 & 19 & 27 & 10 & 10 & 20 & 30 \\
Cutting man hour & 2 & 2 & 4 & 4 & & & & \\
accessory & 1 & 1 & 3 & 3 & & & & \\
The total cost & 3840 & 4170 & 7470 & 10110 & 3130 & 3210 & 6530 & 9420
\end{tabular}

In terms of cost optimization, the average saving rate of the safety barrier in the Volvo workshop is more than 19 percent. As the production of the protective fence increases, the price of the fence and column costs will fall further, the price of non-standard items will rise further, depending on the parts used in different occasions, non-standard and cut working hours will increase further, causing material and artificial consumption.

\section{Fence design}

In this paper, a new design scheme for the optimal design of security fence is proposed. In the case of fence type, opening position, arrangement, etc, by the arrangement of the different number of fences, reduce the length of the site and reduce the cost of protecting the fence, for the special enclosures that need to be cut, the position of the open hole should not be placed on the vertical column, so as not to reduce the strength of the fence and the damage of the fence. The optimized design arrangement can reduce the cutting frequency and non-standard parts use quantity, save labor cost, reduce the cost of protective fence.

\section{References}

[1] Ping Wu. CYJ5-1.8-18HPF Analysis of safety hazard of safety guard of type 5 pumping unit[J]. Security technology,J. 2008,8(10): 105-112

[2] Yuanke Li. Engineering optimization design [M]. Tsinghua university press,2006

[3] Xianfen Lu. Optimization design guide [M]. 2010,(2): 13-14

[4] Jing Chen. Application of mechanical reliability optimization design [J]. Coal technology, 2010.10

[5] Wanxiang Li. Engineering optimization design and MATLAB implementation [M]. Tsinghua university press, 2010.2: 4-6

[6] Emilia Silvas. Theo Hofman. Maarten Steinbuch. Review of Optimal Design Strategies for Hybrid Electric Vehicles. 2012 Workshop on Engine and Powertrain Control, Simulation and Modeling International Federation of Automatic Control Rueil-Malmaison, 2012: 57-64

[7] Jizhao Liang, The design of food mechanical optimization [M]. Chemical industry press, 2009,1: $131-132$

[8] Yanchun Pan. Based on Arena's workshop scheduling problem modeling method and its simulation optimization system design [J]. Computer integrated self-built system. 2006,12(3): 391-394

[9] XinLiu. Based on airbag cushioning, a manned airborne crew protection device optimization design[J]. Journal of mechanical engineering. 2012,11(21): 168-174 
[10] S. Kholopova, Mikhail I. Balzannikova, Vadim Yu. Alpatova, Alexey.V. Solovieva. Girders of hydraulic gates optimal design. Proced Engineering 2016: 277-282 